\title{
Acute Pulmonary Edema of Non-Cardiac Origin Causing Cardiopulmonary Arrest Following Injection of Non-Ionic Intravenous Computed Tomography Scan Contrast: A Case Report and Brief Review
}

\author{
Kuldeep Singh
}

\begin{abstract}
Non-cardiac pulmonary edema following intravenous (IV) contrast medium is a rare form of severe allergic response; however, association of cardiopulmonary arrest with it is even an extremely uncommon occurrence. It is a very serious and life threatening reaction, and it must be immediately recognized and promptly treated to avoid poor outcome. I, report a case of a 77-year-old female who developed an immediate acute pulmonary edema of non-cardiac origin resulting in cardiopulmonary arrest on computed tomography (CT) scan table following IV non-ionic contrast media injection of Optiray 320 during spiral CT of abdomen for a routine outpatient urological disorder for follow-up with a miraculous outcome. There was no known previous reaction or allergy to IV contrast. A prolonged cardiopulmonary resuscitation (CPR) more than $4 \mathrm{~h}$ was required to revive and stabilize the patient's critical condition with complete recovery without any sequel. Take home message is to be familiar with and recognize these catastrophic complications of IV contrast and be prepared to manage them appropriately and immediately to save the patients' life. A routine use of IV Benadryl prior to contrast injection may be beneficial in high risk patients. Due to serious untoward reactions of IV contrast, it may be prudent to discover or invent a new inert substance or improved CT scanning techniques without any contrast. "Thou shall not harm".
\end{abstract}

Keywords: Allergic reactions; Intravenous contrast; Ionic and nonionic contrast media; Non-cardiac pulmonary edema; Cardiac arrest; CPR; Sequel

\section{Introduction}

Adverse and allergic reactions to intravenous (IV) contrast [1] are not uncommon, reactions could vary from a very mild

Manuscript submitted November 2, 2017, accepted November 16, 2017

Department of Emergency Medicine, Twinsburg Ahuja Medical Center, University Hospitals, Twinsburg, OH, USA. Email: drsinghmd@yahoo.com

doi: https://doi.org/10.14740/jmc2955e
$(3-13 \%)[2,3]$ feeling of warmth, nausea or itching to a very serious and life threatening anaphylaxis, anaphylactoid reactions, pulmonary edema [4-6], coronary spasm [7] and cardiac arrest may occur $(0.04-0.3 \%)[8,9]$. Pulmonary edema and cardiac arrest are very rare form of allergic reactions but has been reported in the literature, a case of non-cardiac pulmonary edema was reported by Borish et al in 1998 [10], hence the radiology computed tomography $(\mathrm{CT})$ technician should be fully aware of these reactions, prompt and early recognition followed by immediate medical intervention remains important for a successful outcome. I report a case of acute non-cardiac pulmonary edema $[11,12]$ leading to cardiopulmonary arrest following injection of non-ionic IV CT contrast media. Following a very aggressive and prolonged resuscitation the patient fully recovered without any long term squeal, a rather miracle!

\section{Case Report}

A 77-year-old Caucasian female presented to a free standing radiology facility in August, 2016 in a small medical center for a routine outpatient $\mathrm{CT}$ urography for follow-up of previously removed urinary bladder cancer in recent past by the urologist. She has been doing very well postop with no known cardiopulmonary problem or previous history of IV CT contrast dye or shell fish allergy. After $143 \mathrm{~mL}$ of Optiray 320, a non-ionic contrast media was injected IV, immediately following that the patient experienced severe nausea, vomiting, short of breath and not feeling well. The X-ray technician immediately approached a nurse in the emergency department which is located next door to the X-ray department. A full time Radiologist is not routinely staffed at this facility. The nurse then summoned the emergency room physician on duty (that happened to be the manuscript author) because the patient was experiencing a very serious IV contrast dye reaction. When the physician arrived in the X-ray department, he discovered that the patient was still on CT scanner table, had turned blue, not breathing, with no pulse, mouth and nose covered with pink frothy material. Cardiopulmonary resuscitation (CPR) was immediately commenced in the radiology department and the patient was wheeled into emergency room for further care. CPR continued, secretions continuously suctioned from mouth and nose, venti- 
lated with $100 \%$ oxygen using bag valve mask device followed by tracheal intubation, and treated for acute anaphylaxis with IV epinephrine, corticosteroids, Benadryl, Pepcid, and glucagon. The patient was also treated for acute pulmonary edema with IV Lasix, morphine, frequent suction and positive pressure ventilation. Copious amount of pink frothy material was exuding from mouth, nose and endotracheal tube like an active volcano; bilateral coarse rales on chest auscultation were heard. CPR continued for more than $4 \mathrm{~h}$ for cardiopulmonary arrest, pulseless electrical activity (PEA), hypotension and pulmonary edema. She continued to have problem of effective, satisfactory oxygenation despite positive pressure ventilation with a ventilator. It was recommended by the critical care physician who accepted the patient at intensive care unit (ICU) of the university hospital that she must be transported under the care of an anesthesiologist with nitrous oxide induction, it was accomplished and the patient was transported by air because the facility where the patient was initially resuscitated is a very small and remote free-standing emergency facility with limited resources. Chest X-ray and chest auscultation confirmed the findings of an acute pulmonary edema. The next morning the patient was successfully extubated, fully alert, awake and oriented with normal neurological examination. But she did not remember anything about her serious reaction resulting in cardiopulmonary arrest following IV contrast administration. But she did remember experiencing nausea, vomiting and not feeling well during her CT scan of abdomen. More than a year later of IV contrast reaction, a personal visit paid by the author with the patient in October, 2017 revealed that she is doing very well with no residual squeal and is free of her urinary bladder cancer. She still could not recall much about her serious contrast dye reaction.

Medications used during CPR: all medications were given IV; epinephrine $1 \mathrm{mg}$ five doses, methylprednisolone $125 \mathrm{mg}$ three doses, sodium bicarbonate $8.4 \% 50 \mathrm{mEq}$ three ampules, Pepcid $20 \mathrm{mg}$, benadryl $25 \mathrm{mg}$, glucagon $1 \mathrm{mg}$, lasix $40 \mathrm{mg}$ three doses, morphine $4 \mathrm{mg}$, fentanyl $50 \mu \mathrm{g}$, succinylcholine $50 \mathrm{mg}$, etomidate two doses of $18 \mathrm{mg}$ and $10 \mathrm{mg}$, versed $2 \mathrm{mg}$, dopamine drip, norepinephrine drip, propofol drip, rocephin 1 $\mathrm{g}$, vancomycin $1 \mathrm{~g}$, and nitrous oxide gas.

Procedures performed included: endotracheal intubation, left femoral central line, Lucas-CPR, Foley to $\mathrm{CD}$, and nasogastric tube.

\section{Discussion}

Reactions related to IV contrast have been discussed in detail by Saljoughian, Pharm D PhD in 2012 [13] (5-8\%) and is classified into three categories: 1) mild- feeling of warm, nausea, mild vomiting and mild itching; these side effects are usually short lived and may or may not require any specific treatment, 2) moderate- persistent vomiting, hives, skin swelling and severe itching that normally requires symptomatic treatment with antihistamines, anti nausea medications and corticosteroids, 3) severe anaphylaxis [14], anaphylactoid reactions [15], pulmonary edema, seizures, and cardiac arrest are rare but need immediate medical intervention. In 1970, Ansell [16] reported severe and life threatening reactions with ionic contrast agents in $0.01-0.02 \%$ of all radiological examinations. But in 1992 , Leiberman [17] reported an incidence of severe but not necessarily fatal, anaphylactoid reactions in $1-2 \%$ of all contrast studies, this 10-fold higher incidence may be due to previous under-reporting of adverse reactions. Allergic reaction to IV contrast may be a pseudo allergic response rather than a true antigen-antibody reaction. These agents directly act on mast cells and lead to release of potent vasoactive and pro-inflammatory mediators (histamine, prostaglandins, leukotriene, and cytokines). Tryptase is a neutral protease concentrated in the secretary granules of mast cells [18]. Its concentration in blood is linearly related to histamine release, concentrations above $20 \mathrm{ng} / \mathrm{mL}$ may be indicative of an anaphylactic or anaphylactoid reaction. Studies have shown that iodine [19] is not the true cause of allergic response. Sea food or shell fish allergies are due to special proteins in this food called tropomyosins. Prawns usually cause most allergic response (0.6-10\%). Sea food allergy and topical iodine allergy may or may not increase the risk of IV contrast allergic response.

Patients with prior reaction to IV contrast definitively are at a higher risk of further reactions in future $(40-60 \%)$ and should be prepped with antihistamines and corticosteroids per standard radiology department protocols of its respective institution [20]. Even then some reactions do occur but may be of milder form.

Patients with other multiple allergies, history of asthma, hyperthyroidism [21], congestive heart failure (CHF), dehydration and elderly with diabetes, myasthenia gravis, sickle cell anemia, and pheochromocytoma [22] are at much higher risk of allergic reactions. Renal evaluation especially in the elderly shall be performed before IV contrast injection to avoid further deterioration of renal insufficiency. True allergic response of antigen-antibody reaction is mediated by immunoglobulin $\mathrm{E}$ (IgE); however, Mita et al [23] detected IgE antibody in 1998 following reaction to a radio contrast medium. Skin testing and small test dose of IV contrast media may not be a true predictor of an advanced or serious allergic response after full dose. Reactions are far less common with non-ionic contrast (3\%) as compared to ionic-contrast (12\%) and also ionic contrasts are more immunogenic and cause more serious allergic reactions. Hence non-ionic contrast is now commonly used. Iodinated contrast media is further classified according to osmolality; ionic media $(1,500 \mathrm{mOsm} / \mathrm{kg})$ has higher osmolality than non-ionic $(300-700 \mathrm{mOsm} / \mathrm{kg})$. These agents are derivatives of tri-iodobenjoic acid. In contrast normal serum osmolality is $290 \mathrm{mOsm} / \mathrm{kg}$. Nitric oxide protects against contrast media reactions as reported in an experimental study of rats by Sendo et al [24] in 2000; this study suggests that pulmonary edema is produced by inhibiting endothelial nitric oxide production and nitro vasodilators that protect against adverse effects of contrast media in rats. Nitrous oxide was used in current case and may have been beneficial in this patient based on this experimental study. Role of nitric oxide was further discussed in another experimental study in rats by Yoshikama et al in 1997 [25]. This study concluded that nitric oxide may play a protective role in acute lung injury induced by platelet activating factor in contrast media reactions. Cardiogenic pulmonary edema is caused by increased pressures in the heart resulting in the left ventricular failure to pump all the blood it receives 
from the lungs causing the veins and capillaries of the lungs to be filled with fluids rather than the air as seen in CHF. It occurs in severe coronary artery disease, cardiomyopathy, heart valve disease and hypertension. Non-cardiac pulmonary edema occurs without increase in the heart pressures. Fluid leaks from capillaries of the lungs, air sacs and fills these sacs with fluid rather than air, this also occurs in adult respiratory distress syndrome, acute severe trauma, high altitude, near drowning, smoke inhalation injury, renal artery stenosis. Detailed management of acute contrast reaction has been discussed in USAF Department of Radiology and Biochemical Imaging especially pulmonary edema with oxygen, lasix, morphine, steroids. Cardiopulmonary monitoring during CT scanning, especially in the elderly or high risk patients may be warranted to recognize and promptly treat the serious allergic response due to the IV contrast. CT technician should be well versed with all aliments of IV contrast reactions.

\section{Conflict of Interest}

The author of this manuscript has no conflict of interest and has received no grants.

\section{Grant Support}

The author has received no grants.

\section{References}

1. Shehadi WH. Contrast media adverse reactions: occurrence, recurrence, and distribution patterns. Radiology. 1982;143(1):11-17.

2. Katayama H, Yamaguchi K, Kozuka T, Takashima T, Seez P, Matsuura K. Adverse reactions to ionic and nonionic contrast media. A report from the Japanese Committee on the Safety of Contrast Media. Radiology. 1990; 175(3):621-628.

3. Lasser EC. Adverse reactions to intravascular administration of contrast media. Allergy. 1981;36(6):369-373.

4. Bristedt P, Tylen U. Pulmonary edema following intravenous injection of nonionic low-osmolar contrast medium - appearance on HRCT. A case report. Acta Radiol. 1998;39(1):81-83.

5. Kozlowski C, Kollef MH. Noncardiogenic pulmonary edema associated with intravenous radiocontrast administration. Chest. 1992;102(2):620-621.

6. Mikkelsen LR. [A case of pulmonary edema after intravenous administration of iohexol (Omnipaque) $300 \mathrm{mg} \mathrm{I} /$ $\mathrm{ml}$ in cerebral CT]. Ugeskr Laeger. 1991;153(36):24952496.

7. Irivbogbe OA, Sayyed R. Acute ST-segment elevation induced by intravenous contrast medium. J Clin Exp Cardiology. 2015;6:360.

8. Kao HW, Wu CJ, Lo CP, Chang WC, Chen CY. Computed tomographic features of circulatory arrest. J Formos
Med Assoc. 2006;105(4):359-362.

9. Ko SF, Ng SH, Chen MC, Lee TY, Huang CC, Wan YL. Sudden cardiac arrest during computed tomography examination: clinical findings and "dense abdominal veins" on computed tomography. J Comput Assist Tomogr. 2003;27(1):93-97.

10. Borish L, Matloff SM, Findlay SR. Radiographic contrast media-induced noncardiogenic pulmonary edema: case report and review of the literature. J Allergy Clin Immunol. 1984;74(1):104-107.

11. Bouachour G, Varache N, Szapiro N, L'Hoste P, Harry $\mathrm{P}$, Alquier P. Noncardiogenic pulmonary edema resulting from intravascular administration of contrast material. AJR Am J Roentgenol. 1991;157(2):255-256.

12. Solomon DR. Anaphylactoid reaction and non-cardiac pulmonary edema following intravenous contrast injection. Am J Emerg Med. 1986;4(2):146-149.

13. Saljoughian M, Pharm D. Intravenous contrast media. A review of allergic reactions. U.S. Pharm. 2012;37(5):HS1.

14. Ewan PW. Anaphylaxis. BMJ. 1998;316(7142):14421445.

15. Laroche D, Namour F, Lefrancois C, Aimone-Gastin I, Romano A, Sainte-Laudy J, Laxenaire MC, et al. Anaphylactoid and anaphylactic reactions to iodinated contrast material. Allergy. 1999;54(Suppl 58):13-16.

16. Ansell G. Adverse reactions to contrast agents. Scope of problem. Invest Radiol. 1970;5(6):374-391.

17. Leiberman P. Anaphylactoid reactions to radio contrast material immunol. Allergy Clinics of North America. 1992;3:649-670.

18. Schwartz LB, Metcalfe DD, Miller JS, Earl H, Sullivan T. Tryptase levels as an indicator of mast-cell activation in systemic anaphylaxis and mastocytosis. N Engl J Med. 1987;316(26):1622-1626.

19. Coakley FV, Panicek DM. Iodine allergy: an oyster without a pearl? AJR Am J Roentgenol. 1997;169:951-952.

20. Greenberger PA, Patterson R, Radin RC. Two pretreatment regimens for high-risk patients receiving radiographic contrast media. J Allergy Clin Immunol. 1984;74(4 Pt 1):540-543.

21. Lorberboym M, Mechanick JI. Accelerated thyrotoxicosis induced by iodinated contrast media in metastatic differentiated thyroid carcinoma. J Nucl Med. 1996;37(9):1532-1535.

22. Mukherjee JJ, Peppercorn PD, Reznek RH, Patel V, Kaltsas G, Besser M, Grossman AB. Pheochromocytoma: effect of nonionic contrast medium in CT on circulating catecholamine levels. Radiology. 1997;202(1):227-231.

23. Mita $\mathrm{H}$, Tadokoro $\mathrm{K}$, Akiyama $\mathrm{K}$. Detection of IgE antibody to a radiocontrast medium. Allergy. 1998;53(12):1133-1140.

24. Sendo T, Kataoka Y, Takeda Y, Furuta W, Oishi R. Nitric oxide protects against contrast media-increased pulmonary vascular permeability in rats. Invest Radiol. 2000;35(8):472-478.

25. Yoshikama, et al. Experimental study in rats. Critical Care Medicine. 1997;25(2):286-292. 